Supplement of Saf. Nucl. Waste Disposal, 1, 125-126, 2021

https://doi.org/10.5194/sand-1-125-2021-supplement

(C) Author(s) 2021. CC BY 4.0 License.

Supplement of

\title{
Discussion of parameters used to distinguish suitable from less suitable HLRW bentonites
}

\author{
Stephan Kaufhold \\ Correspondence to: Stephan Kaufhold (s.kaufhold@bgr.de)
}

The copyright of individual parts of the supplement might differ from the article licence. 


\section{Discussion of parameters which can be used to distinguish suitable from less suitable HLRW bentonites}

Bentonite will be used as geotechnical barrier mostly in crystalline rocks

Bentonite:

backfill + blocks

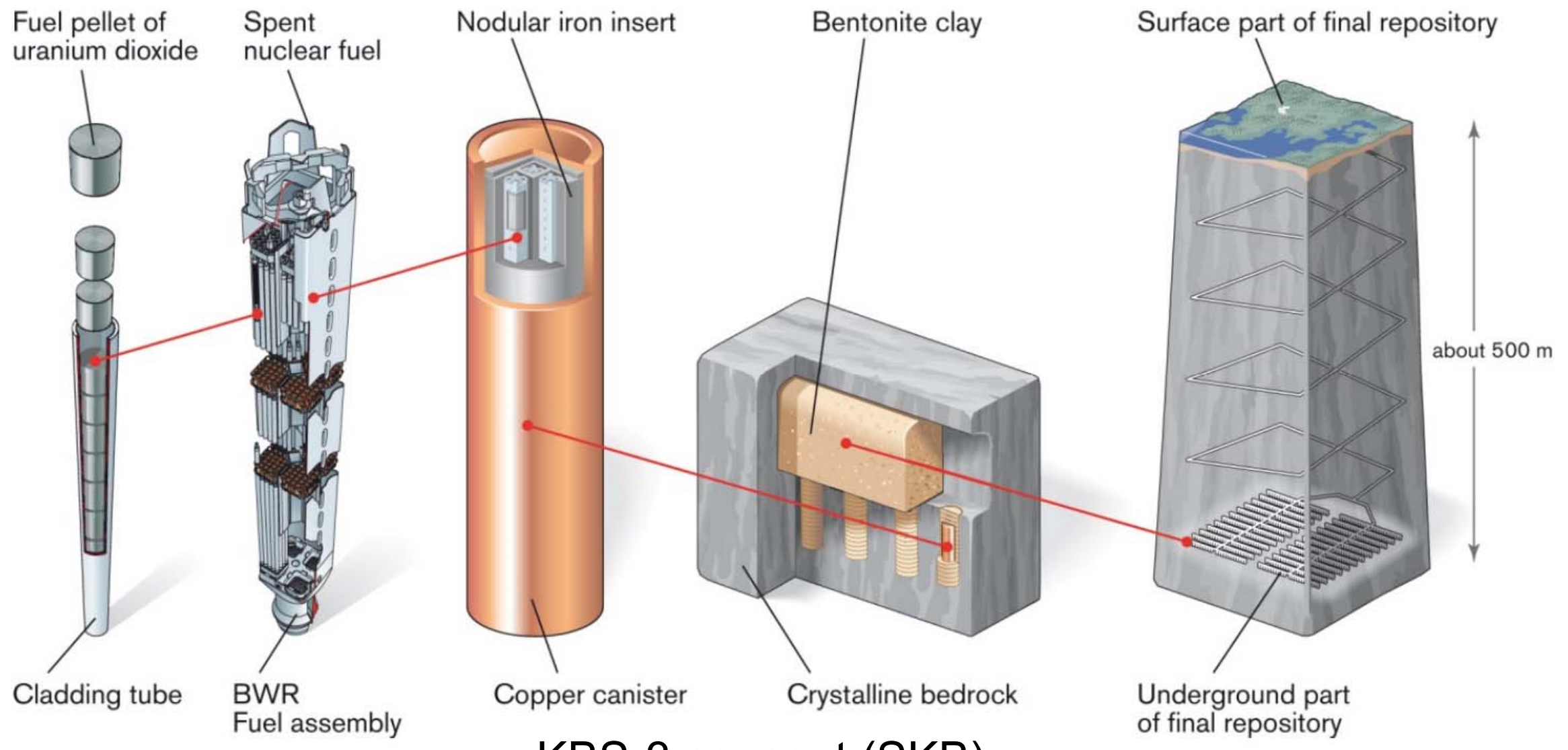

KBS-3 concept (SKB) 
Discussion of parameters which can be used to distinguish suitable from less suitable HLRW bentonites

Most relevant: geotechnical barrier

(compacted bentonite blocks to seal canister from crystalline rock)
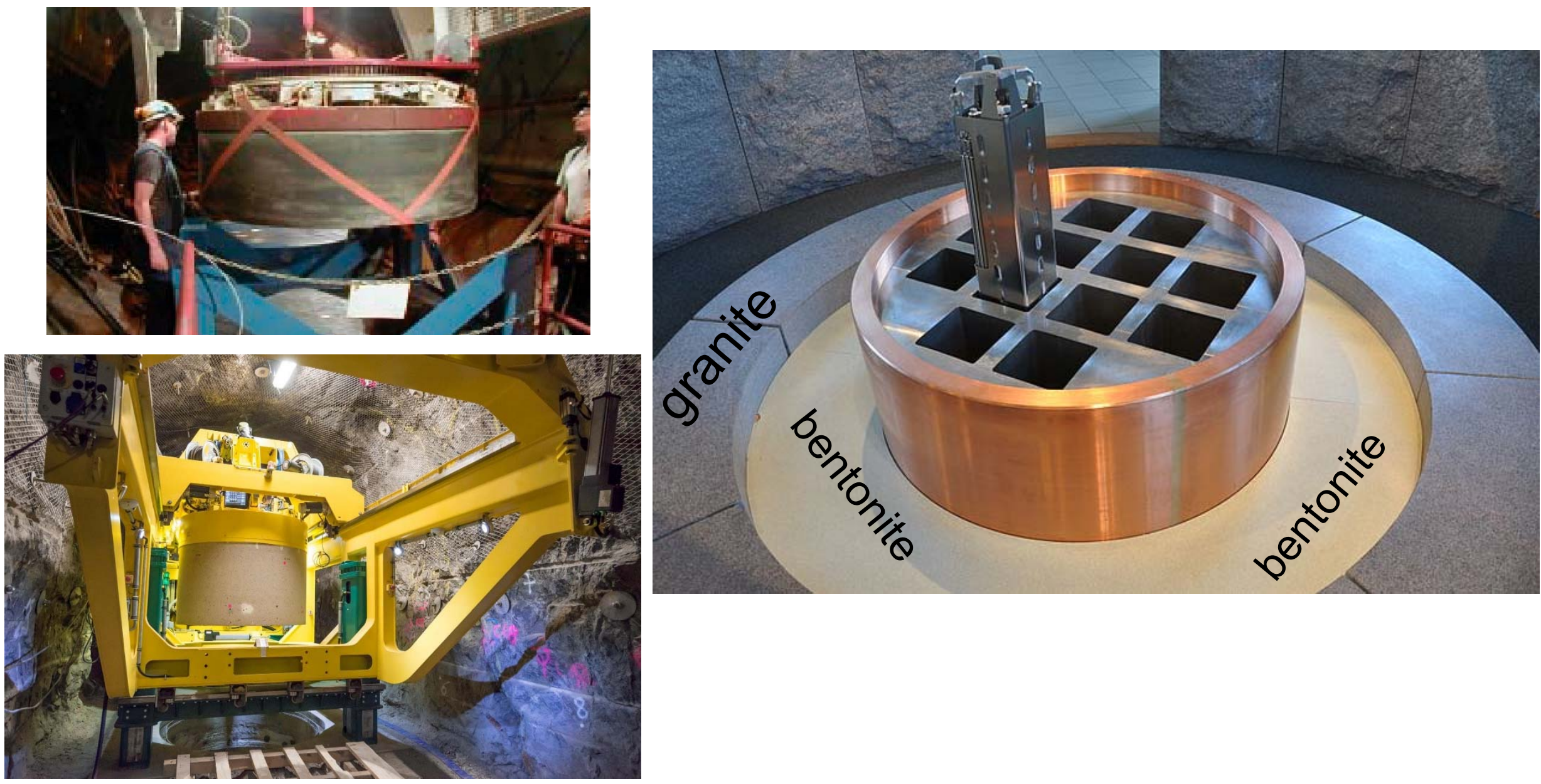
Discussion of parameters which can be used to distinguish suitable from less suitable HLRW bentonites

Why bentonite?

Bentonite $=$ swelling clay $=$ low hydraulic conductivity since decades: sealing of landfills, contaminated sites, groundwater,...

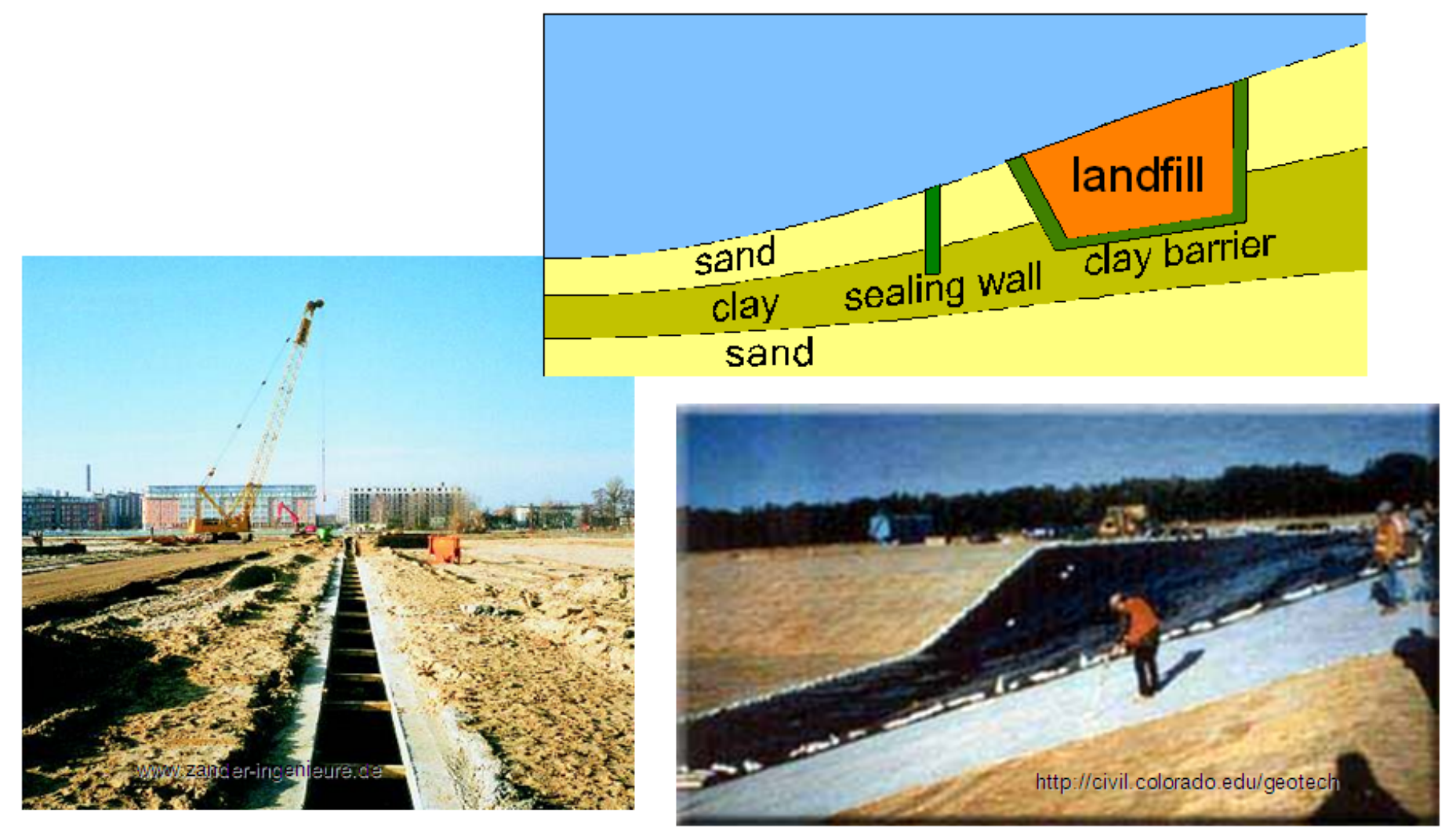


Discussion of parameters which can be used to distinguish suitable from less suitable HLRW bentonites

SKB started to systematically investigate bentonite as possible HLRW-barrier more than 40 years ago!

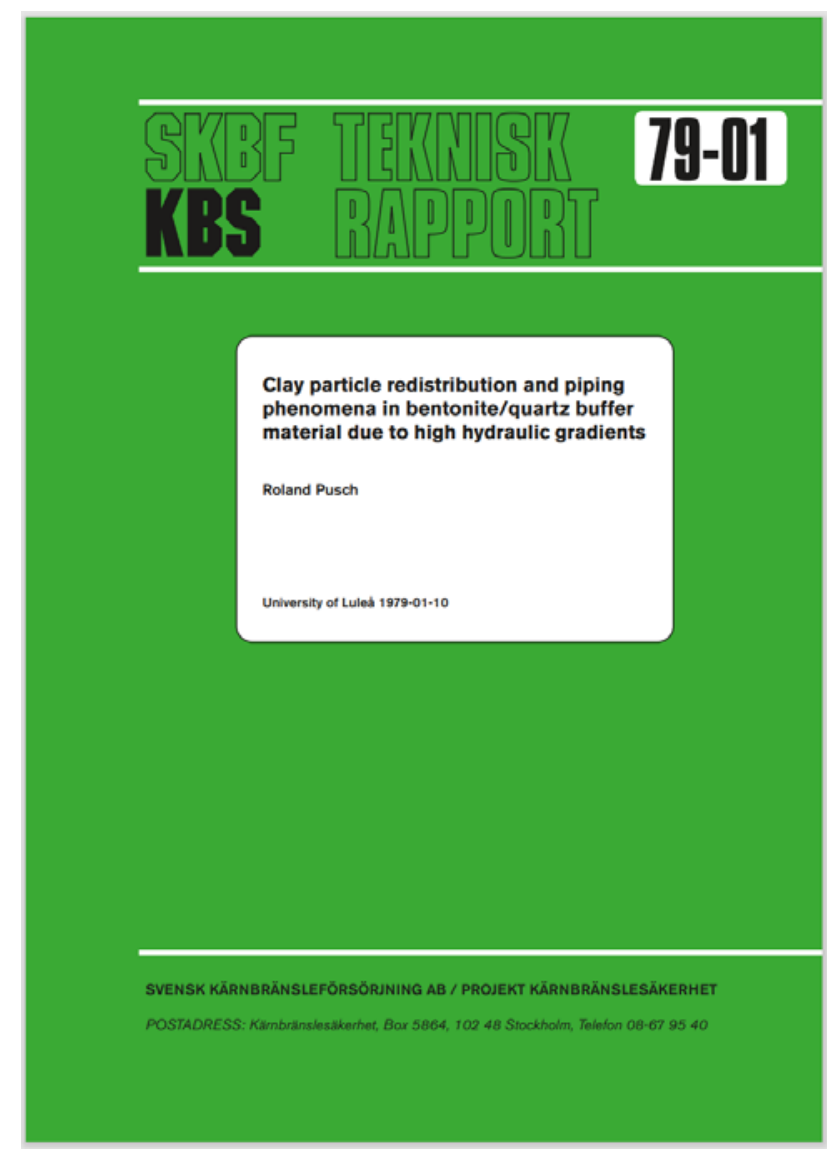

...and the possible problems / challenges of this new application 


\section{Discussion of parameters which can be used to distinguish suitable from less suitable HLRW bentonites}

Desired / required properties of the bentonite barrier?

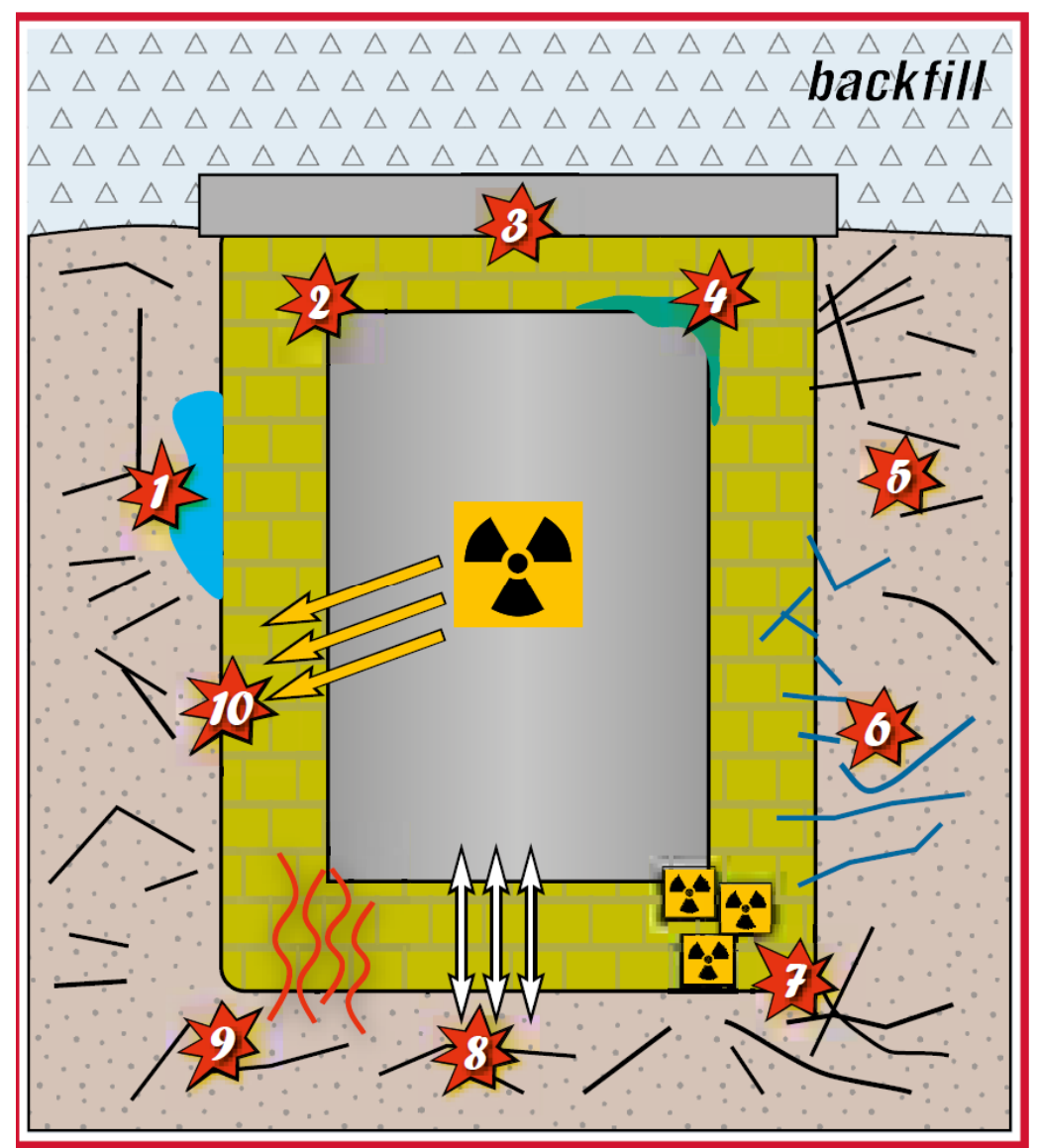

1 Sealing

(swelling capacity)

2 Stability: Drying (T)

3 Stability: Cement water

4 Corrosion

5 Erosion

6 Stability: Salt solutions

7 Retention

(radionuclide adsorption)

8 Canister displacement

9 Thermal conductivity

10 Stability: Radiation

Kaufhold, S. ed. (2021) Bentonites - from mine to application. - Geologisches Jahrbuch B 107, Schweizerbart, ISBN 978-3-510-96859-6. Online accessible:

https://doi.org/10.1127/bentonites/9783510968596 
Discussion of parameters which can be used to distinguish suitable from less suitable HLRW bentonites

Problem: Bentonite is a highly variable natural material !!

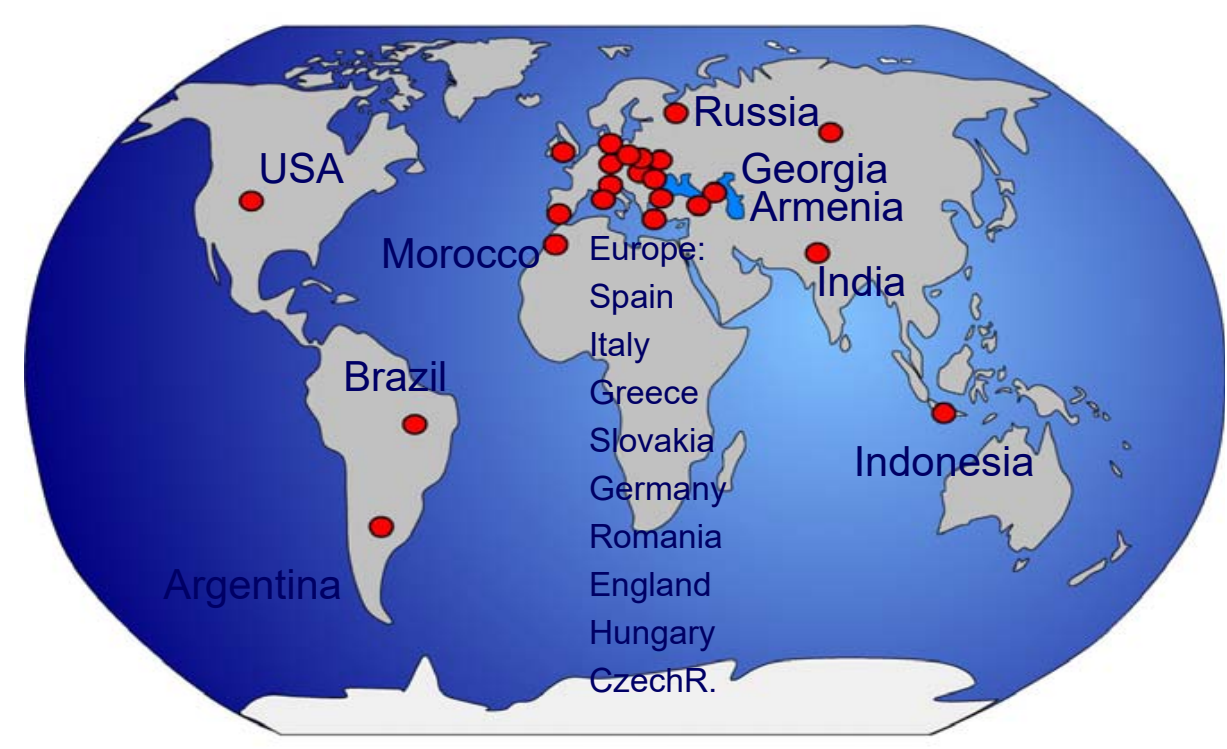

BGR:

Comparison of 38 different bentonite samples from all over the world

+ large scale tests

ABM, LOT, PTR, FEBEX, HotBent, ...
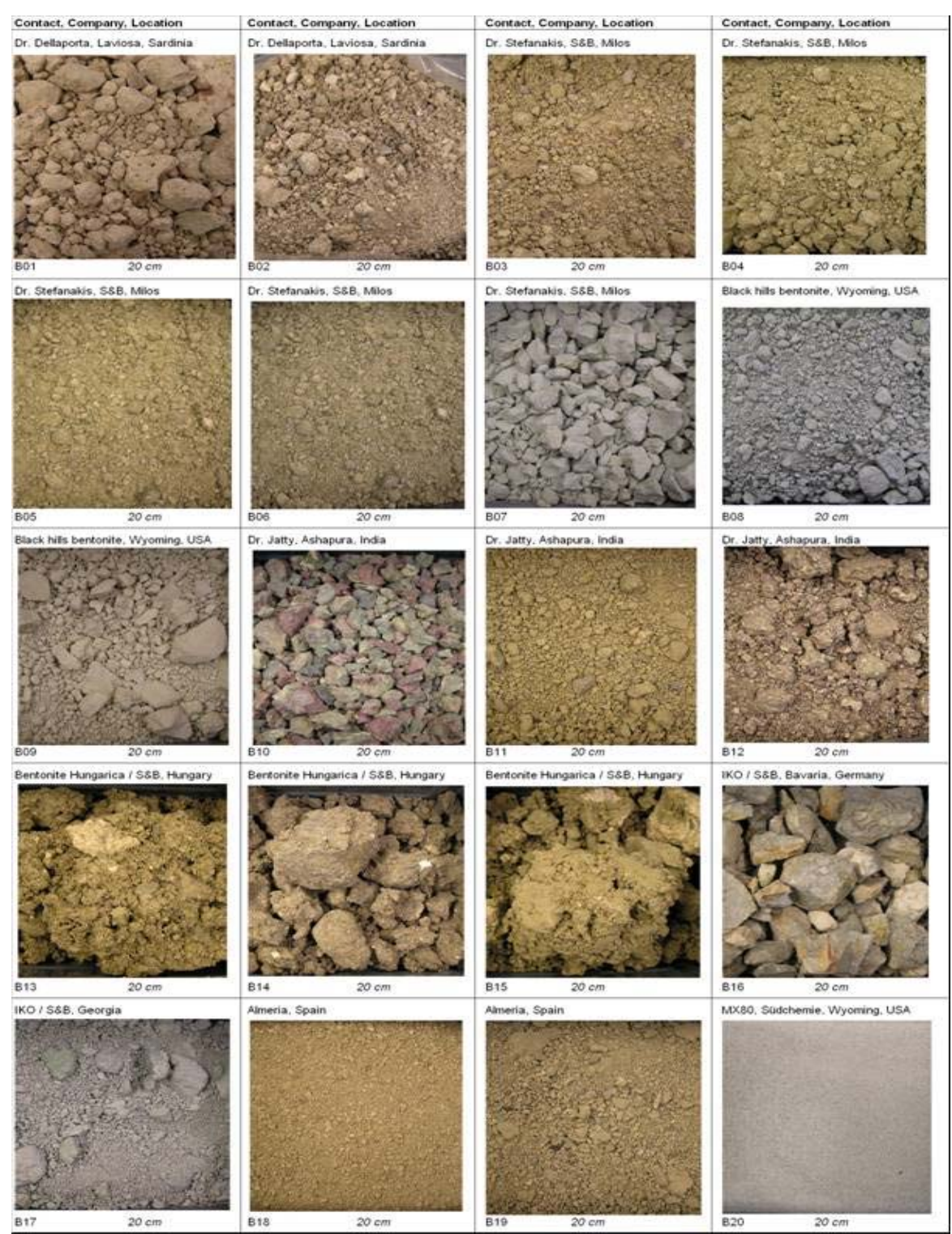

Dr. S. Kaufhold 


\section{Discussion of parameters which can be used to distinguish suitable from less suitable HLRW bentonites}

Which bentonite is most suitable??

Some properties depend more on compaction than on type of material (no suitable criteria for bentonite selection)

Higher compaction:

higher dry density

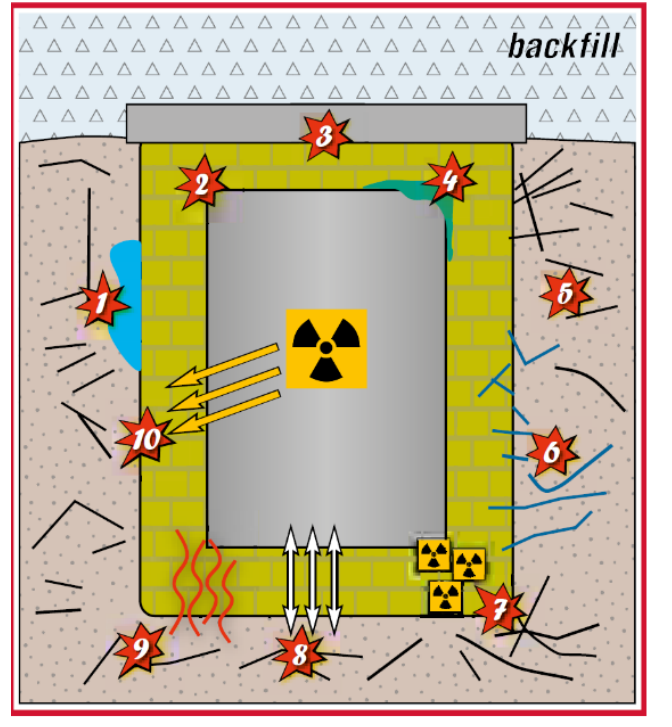

Stability: Drying ( $\mathrm{T}$ )

Stability: Cement water

Corrosion

Erosion

Stability: Salt solutions

Retention

(radionuclide adsorption)

+ lower hydraulic conductivity (sealing)

+ less canister displacement

+ higher thermal conductivity 


\section{Discussion of parameters which can be used to distinguish} suitable from less suitable HLRW bentonites

Stability Drying

High temperature $=$ cation fixation $=$ loss of swelling capacity

not specific for different bentonites Kaufhold \& Dohrmann (2010)
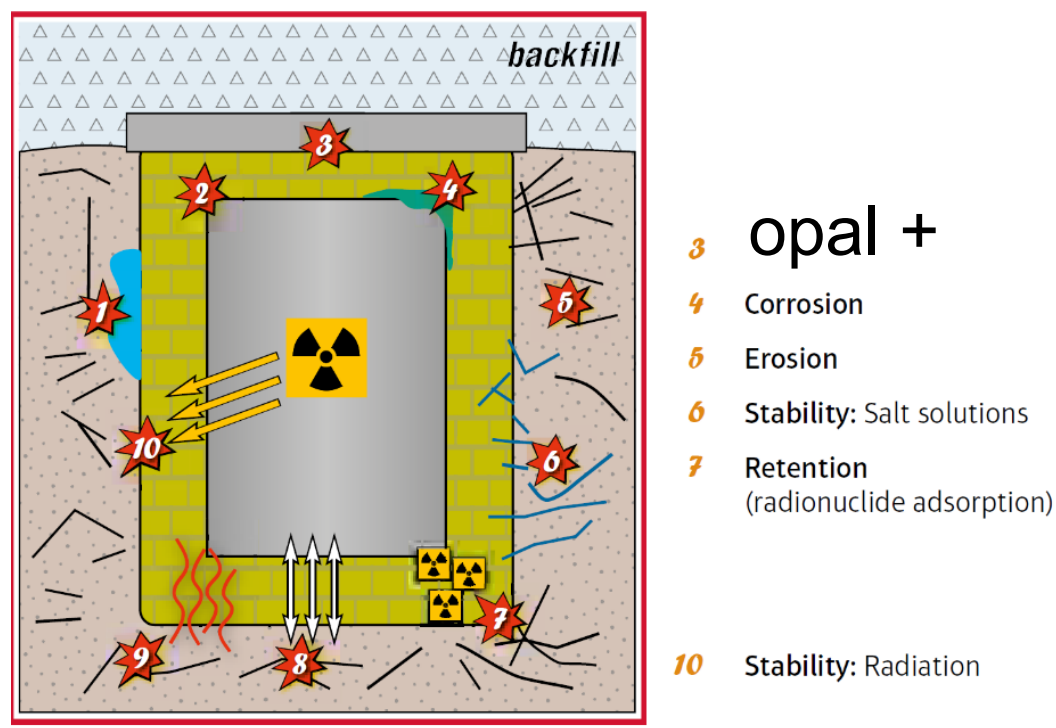

Stability Cement (Kaufhold et al., 2020)

Highly alkaline cement solutions dissolve bentonite

Reactive silica (opal) present in some bentonites buffers the $\mathrm{pH}$ 


\section{Discussion of parameters which can be used to distinguish}

suitable from less suitable HLRW bentonites

Corrosion $(\mathrm{Fe})$

Kaufhold et al. (2015)

Low charge $=$ high corrosion

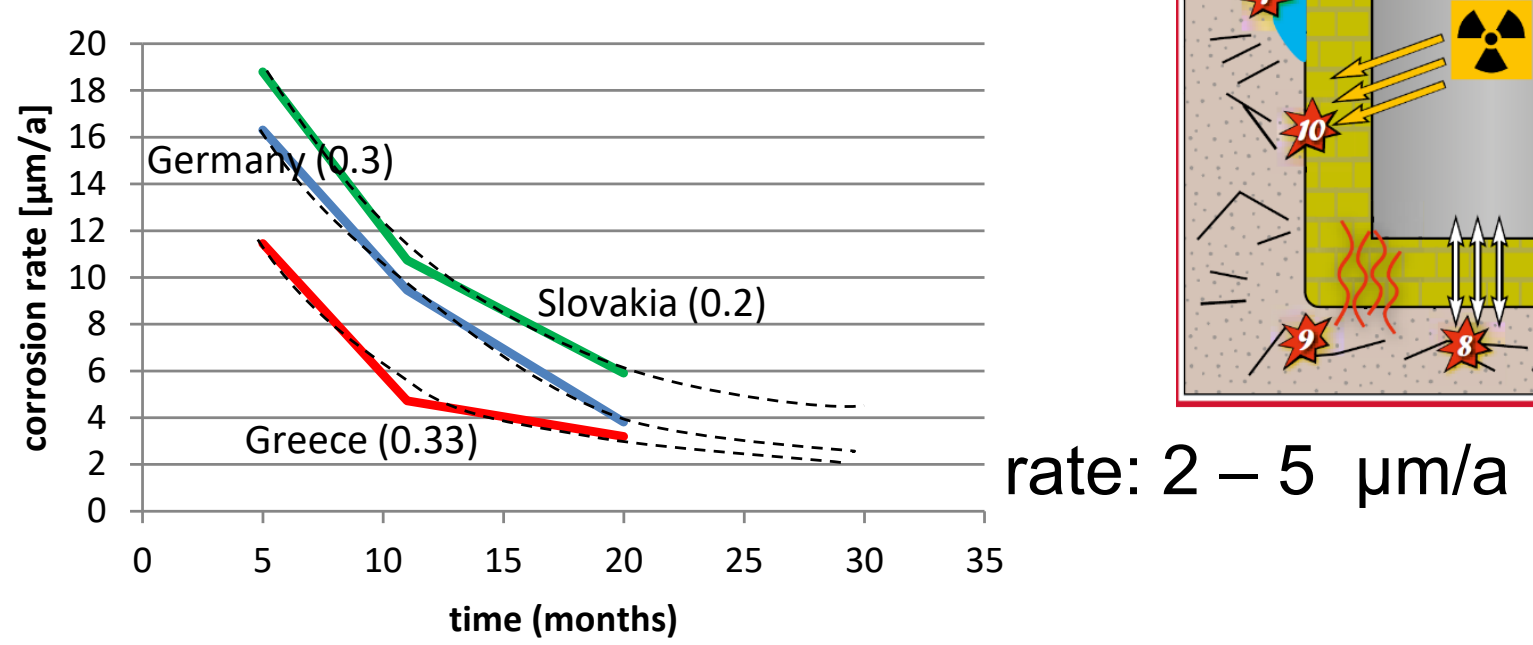

Corrosion (Cu) Kaufhold \& Dohrmann (2017): only pyrite interacts with copper

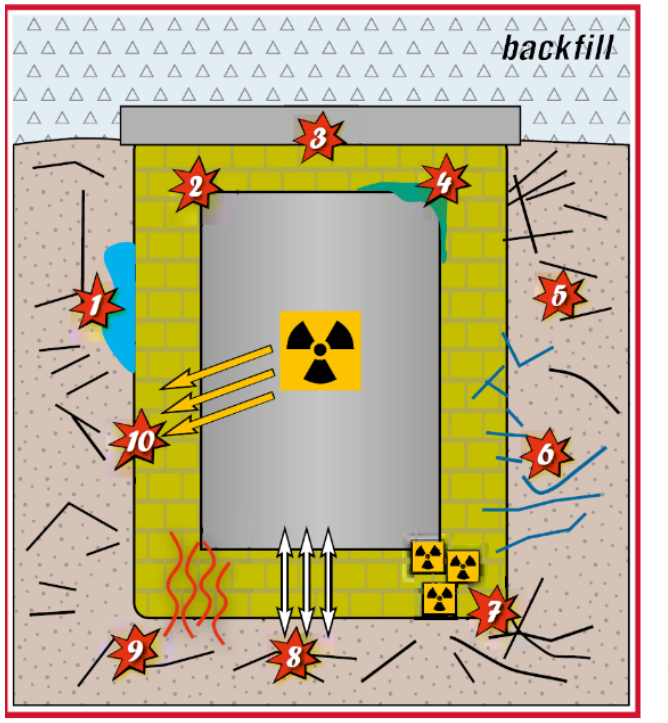

opal + pyrite charge + Erosion Stability: Salt solutions Retention (radionuclide adsorption)

10 Stability: Radiation

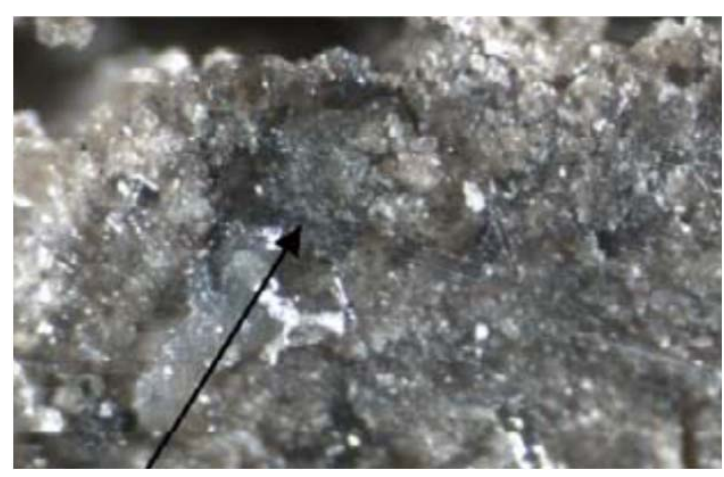

Kaufhold, S., Sanders, D., Dohrmann, R., Hassel, A.-W. (2015) Fe corrosion in contact with bentonites. - Journal of Hazardous Materials, 285, 464-473.

Kaufhold, S., Dohrmann, R., Gröger-Trampe, J. (2017) Reaction of native copper in contact with pyrite and bentonite in anaerobic water at elevated temperature. - Corr. Eng. Sci. and Tech., 52, 349 - 358 
Discussion of parameters which can be used to distinguish suitable from less suitable HLRW bentonites

\section{Erosion}

Kaufhold Dohrmann (2008)

High $\mathrm{Na}$-content $=$ high erosion

Kaufhold et al. (2021)

Cation exchange is fast and cannot be avoided

$=>$ No reasonable criteria

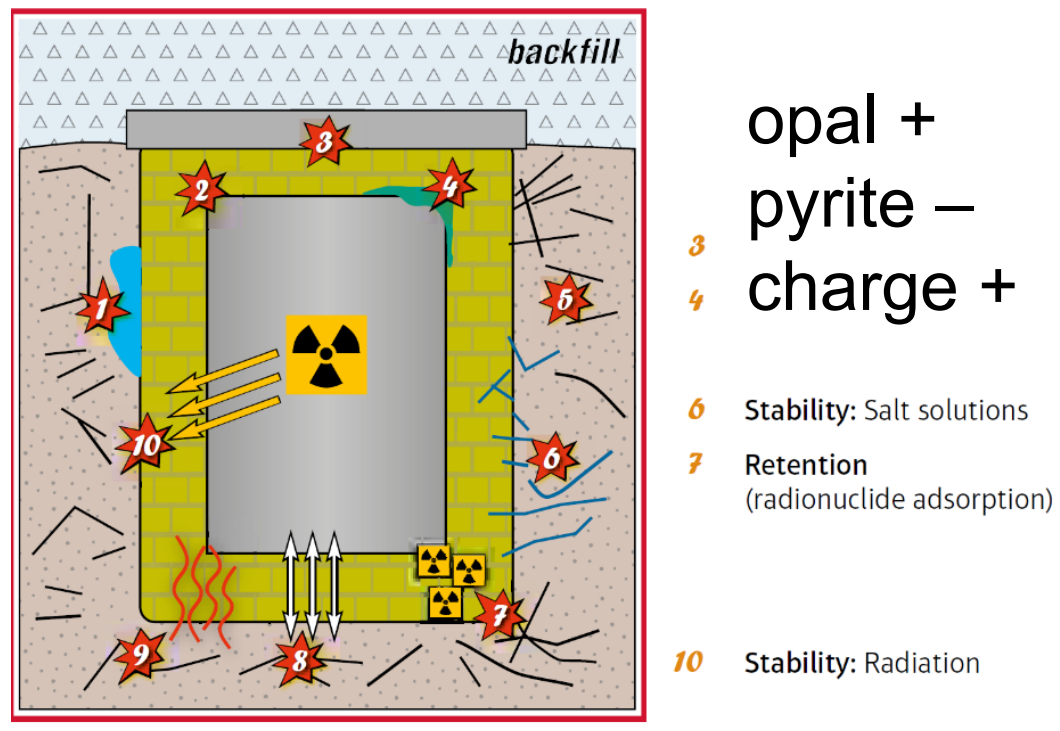




\section{Discussion of parameters which can be used to distinguish}

suitable from less suitable HLRW bentonites

Stability salt solutions

Kaufhold \& Dohrmann $(2009,2010)$, Kaufhold et al. (2019)

Complex interaction with clay minerals - poorly understood...

Reactive (partly soluble) phases should be absent

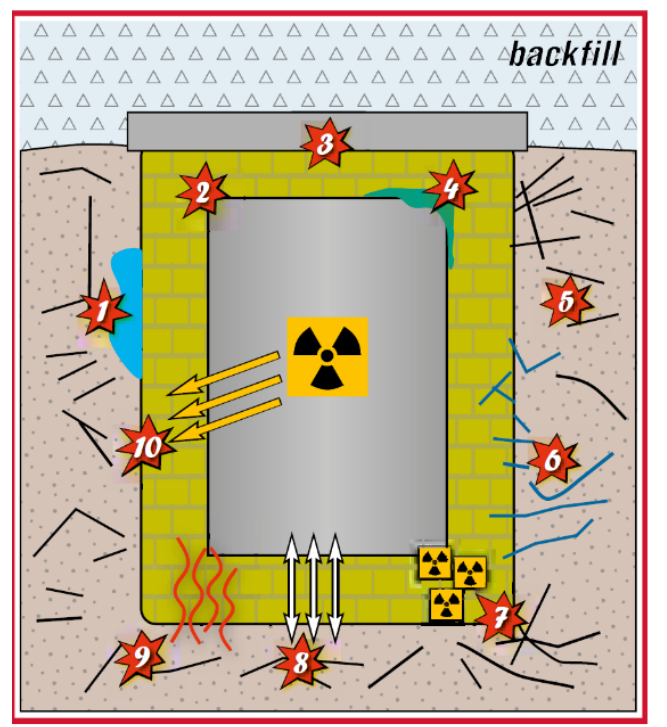
opal + pyrite charge + cal/gyp om -

No pyrite, no calcite, no gypsum, no organic matter 


\section{Discussion of parameters which can be used to distinguish}

suitable from less suitable HLRW bentonites

Radionuclide adsorption

Different radionuclides have different properties, bentonites cannot adsorb all

$=>$ focus on the most hazardous?

$I^{129}$ is considered one of the most hazardous - not retained by pure bentonite

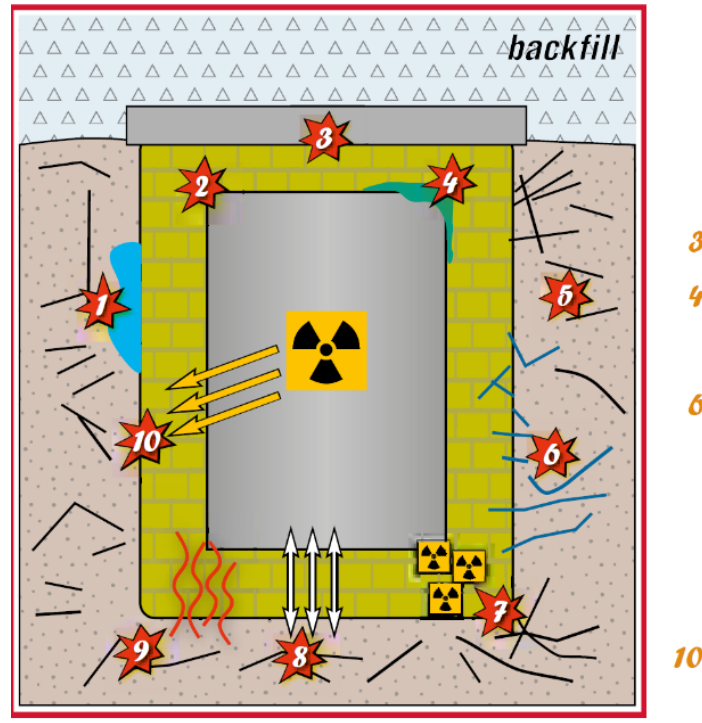

opal + pyrite charge + cal/gyp om -

Bentonite can be modified with respect to adsorption of specific radionuclides (Kaufhold et al., 2007), but no generally applicable criteria 
Discussion of parameters which can be used to distinguish suitable from less suitable HLRW bentonites

Stability against radiation

Fe rich bentonites are more affected by radiation

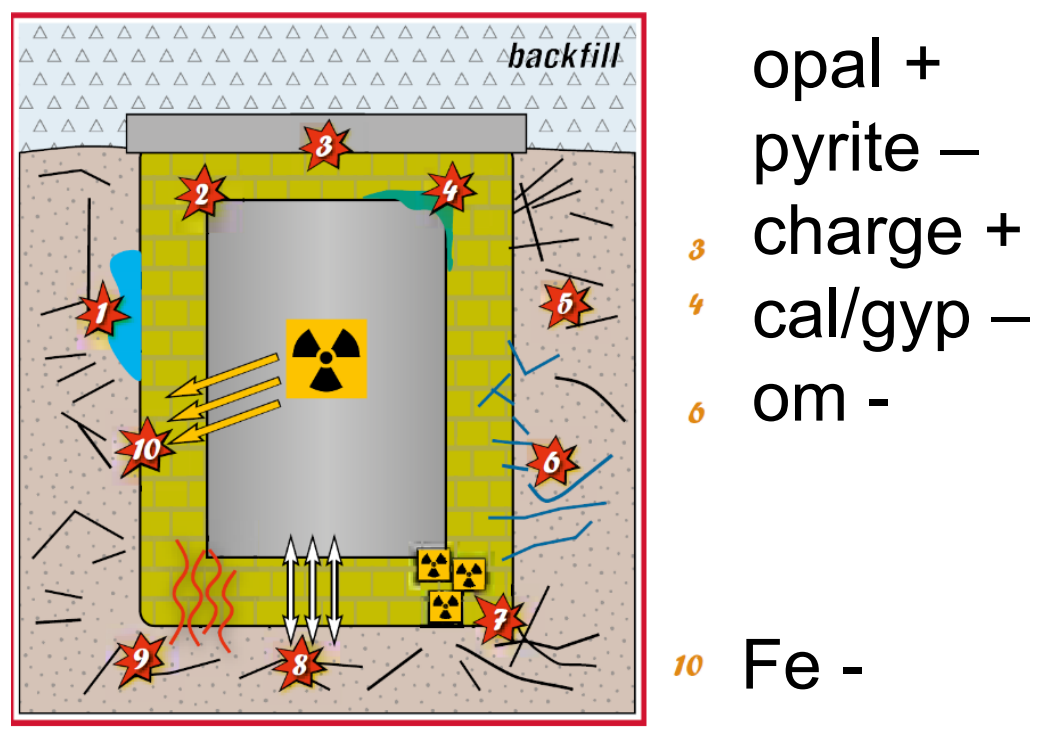




\section{Discussion of parameters which can be used to distinguish suitable from less suitable HLRW bentonites}

Selection of suitable bentonites

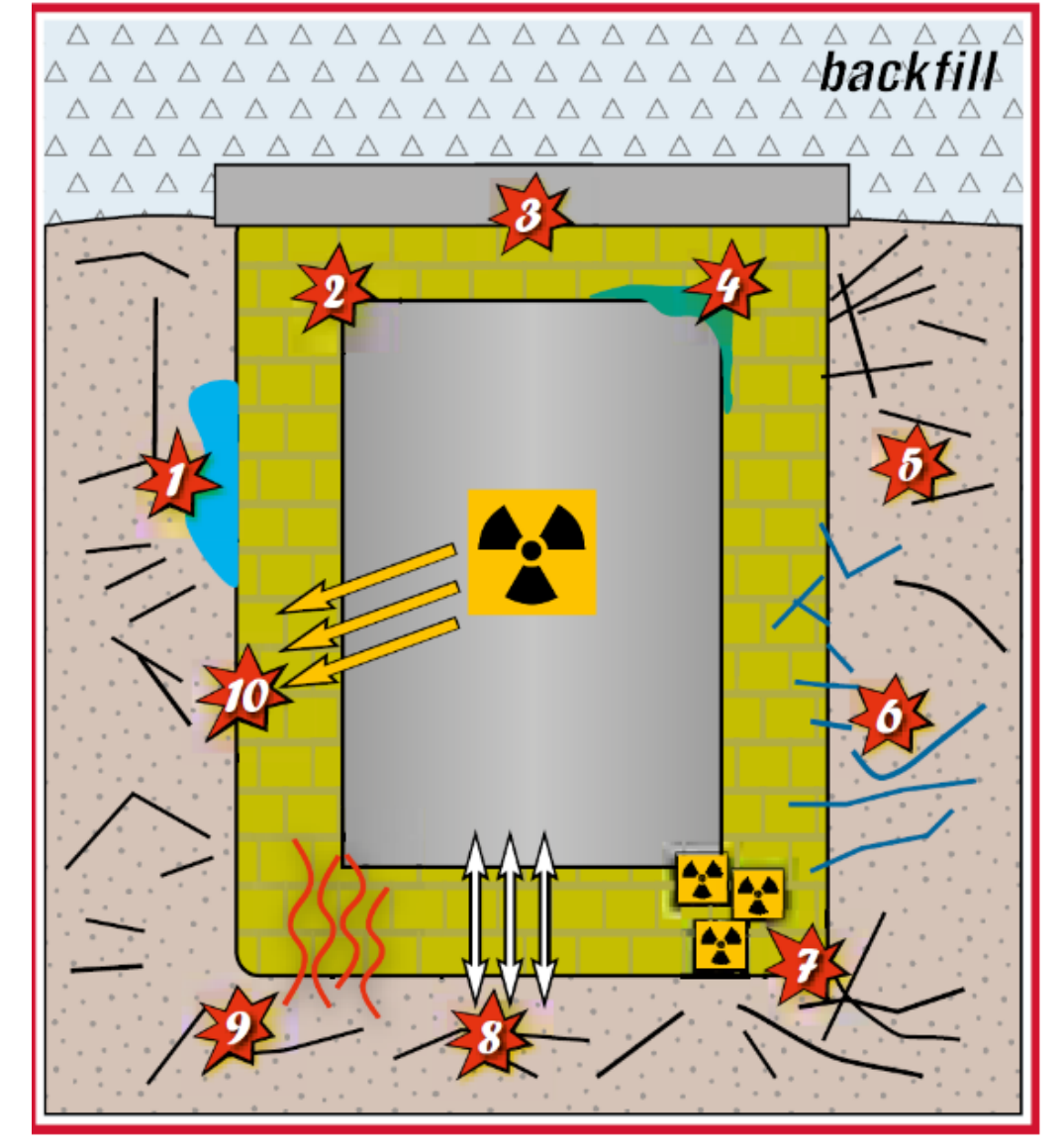

\section{Positive}

3 Stability: Cement water

presence of some opal

4 Corrosion

low pyrite, high charge presence of opal?carb?

6 Stability: Salt solutions low pyrite,

low calcite low gypsum low organic matter

10 Stability: Radiation

low $\mathrm{Fe}$

Currently: use domestic bentonite or buy MX80 (Wyoming bentonite)

No bentonite selection decision taken yet (even not Posiva) 


\section{thanks}

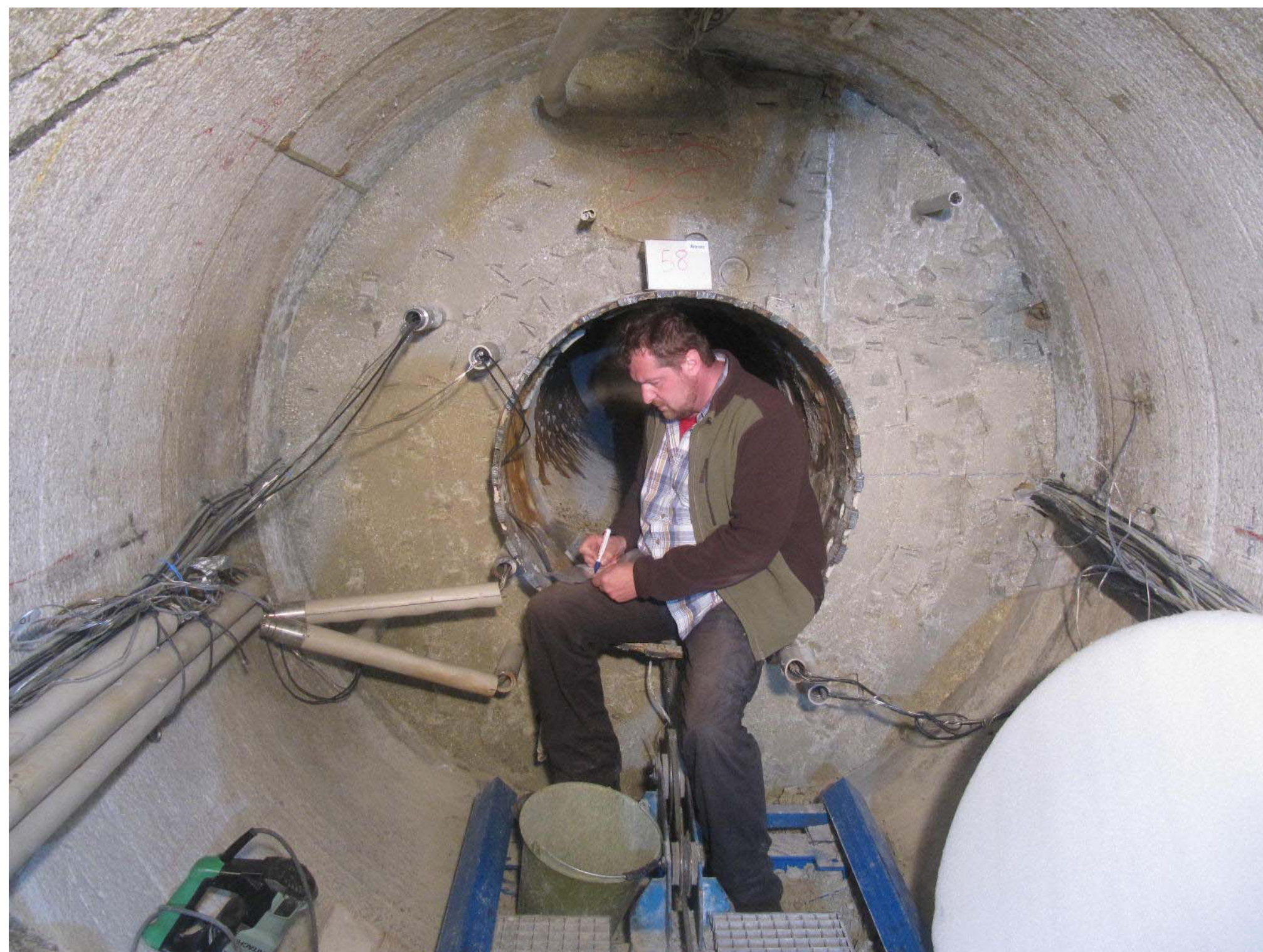

